\title{
Oral health status of women with high-risk pregnancies
}

\author{
Vlasta Merglova ${ }^{a}$, Hana Hecova ${ }^{a}$, Jaroslava Stehlikova ${ }^{a}$, Pavel Chaloupka ${ }^{b}$
}

\begin{abstract}
Aim. The aim of this study was to investigate the oral health status of women with high-risk pregnancies.
Methods. A case-control study of 142 pregnant women was conducted. The case group included 81 pregnant women with high-risk pregnancies, while 61 women with normal pregnancies served as controls. The following variables were recorded for each woman: age, general health status, DMF, CPITN, and PBI index, amounts of Streptococcus mutans in the saliva and dental treatment needs. The Mann-Whitney test, Kruskal-Wallis test, t-test and chi-squared test were used for statistical analyses.

Results. Statistically significant differences were detected between the PBI indices and dental treatment needs of the two groups. Out of the entire study cohort, $77 \%$ of the women in the case group and $52 \%$ of the women in the control group required dental treatment.
\end{abstract}

Conclusion. In this study, women with complications during pregnancy had severe gingivitis and needed more frequent dental treatment than those in the control group.

Key words: normal pregnancy, high-risk pregnancy, dental caries, periodontal disease, Streptococcus mutans

Received: November 15, 2011; Accepted: April 12, 2012; Available online: June 1, 2012

http://dx.doi.org/10.5507/bp.2012.045

aDentistry Department, Faculty of Medicine in Pilsen, Charles University in Prague, Czech Republic

${ }^{b}$ Gynaecological and Obstetrics Department, Faculty of Medicine in Pilsen, Charles University in Prague

Corresponding author: Vlasta Merglova, e-mail: merglovav@fnplzen.cz

\section{INTRODUCTION}

Pregnancy is accompanied by changes in the oral cavity that affect the hard and soft tissues of the mouth ${ }^{1}$. Most pregnant women change their eating habits to more frequently eat foods rich in carbohydrates and acids. This situation is exacerbated by the decrease in salivary $\mathrm{pH}$ associated with frequent nausea and vomiting. Pregnant women who do not comply with regular and careful oral hygiene often suffer from erosions of tooth enamel and develop new dental caries. In dental decay etiology, we observe a key role played by the microorganisms present in dental plaque. The most important microorganisms with regard to dental decay are viridans streptococci, especially Streptococcus mutans (SM), which can be transmitted by the mother's saliva to the child's oral cavity. A high level of SM in the saliva of a pregnant woman is considered a risk factor for early infection of her child's oral cavity and the development of early childhood caries ${ }^{1,2}$.

Hormonal changes associated with pregnancy, together with poor oral hygiene, are responsible for the development of gingivitis (gingivitis gravidarum). Swelling, redness and bleeding from the gums can appear as early as the second trimester and peak in the eighth month of pregnancy. Gingivitis is more common in pregnant women who have existing pre-pregnancy gum problems because they failed to follow regular and careful oral hygiene practices prior to becoming pregnant. Untreated gingivitis can cause periodontitis, which can correlate with preterm delivery, low birth weight, early pregnancy loss, preeclampsia and chorioamnionitis ${ }^{1,3,4}$.
Premature labor and low birth weight can be caused by a number of complications during pregnancy ${ }^{1}$. Women with certain general health problems (e.g., heart diseases, high blood pressure, renal diseases, autoimmune disorders, sexually transmitted diseases, diabetes mellitus and cancer), those with multiple pregnancies or previous highrisk pregnancies, those becoming pregnant over the age of 35 and those with gynecological problems (e.g., menstrual disorders, uterine developmental anomalies and infertility problems) experience high-risk pregnancies more frequently than the general population.

Offenbacher et al. ${ }^{5}$ first suggested that poor oral health could cause pregnancy complications, and later studies ${ }^{6.8}$ have reached the same conclusions. Another study, however, has found no association between poor dental health and pregnancy complications ${ }^{9}$. The present study investigates whether associations can be found between recorded oral health variables in women with normal pregnancies and women with high-risk pregnancies.

We hypothesized that statistically significant differences in selected oral health indicators would exist between the two groups.

\section{MATERIALS AND METHODS}

\section{Selection of pregnant women}

This study was part of a long-term research project supported by the Health Ministry of the Czech Republic. It was performed at the Gynecological and Obstetric Department and the Dentistry Department of 
the University Hospital in Pilsen, the Czech Republic. In March, April and May of 2010, all the women in their third trimester of pregnancy who attended the Gynaecological and Obstetric Department for an antenatal check up were offered a clinical examination at the Dentistry Department. The pregnant women were informed about the study, and 142 of them agreed to participate in the study and provided written consent. The study group comprised Caucasian women with homogeneous ethnic backgrounds and a range of socio-economic statuses.

Prior to the clinical dental examination, the pregnant women were divided into two groups: the research group $\mathrm{R}$ comprised women with high-risk pregnancies, defined as those with the presence of any systemic diseases, gynecological complications, age-related risk factors or those who used in vitro fertilization (IVF) $(\mathrm{N}=81)$; the control group $\mathrm{F}$ comprised women with normal pregnancies $(\mathrm{N}=61)$.

\section{Clinical dental examination}

The dental examinations were conducted under standardized conditions using a dental chair, artificial light, a dental mirror and dental and periodontal probes in a dentist's office. The dental examinations were performed by two experienced teachers of the Dentistry Department who agreed on the criteria for the detection of dental caries and periodontal problems prior to the examinations.

The following variables were recorded for each woman: anamnestic data (age and general health status), Decayed, Missing, Filled index (DMF), the Papilla Bleeding Index (PBI), the Community Periodontal Index of Treatment Needs (CPITN), amount of SM present in the saliva and dental treatment needs. Dental caries was recorded_according to WHO criteria ${ }^{10}$; all tooth surfaces were visually examined. The dental probe was used only to remove debris and food particles in order to improve visibility. Caries was recorded as present when a lesion in a pit or fissure or on a smooth tooth surface had an unmistakable cavity, undermined enamel or a detectably softened floor or wall.

The DMF index is determined as the sum of D + M + F. D (decay) is the number of teeth with untreated dental decay, M (missing) is the number of teeth that were extracted due to decay, and F (filled)is the number of fillings or crowns that were placed due to dental caries.

The PBI index is an indicator of gingival inflammation severity because one of the major symptoms of gingivitis is bleeding upon probing. The degree of gingival bleeding was assessed on the basis of irritation: value 0 , no bleeding; value 1 , bleeding point appears on the edge of the investigated papilla; value 2 , fine blood line or a few bleeding points appear on the edge of the investigated papilla; value 3, interdental space is filled with blood; and value 4 , bleeding occurs immediately after irritation, and a drop of blood flows to the surroundings. We evaluated the gingival papillae of seven teeth in each quadrant. Papillae were investigated in the upper right quadrant palatally, upper left buccally, lower right buccally and left lingually.
The maximum and mean values were recorded for each subject.

The CPITN index is recommended for use in adults aged 20 years and older. A specially designed periodontal probe is used to determine the presence or absence of bleeding, calculus, defective margins of restorations and pathological periodontal pockets. We investigated CPITN in four lateral and two frontal sextants in the jaw. The following tooth sites were probed: the upper jaw mesiobuccal, buccal and distobuccal and the lower jaw mesiolingual, lingual and distolingual. The most severe finding of each examined tooth was recorded. The values were assessed as follows: value 0 , healthy periodontal tissues; value 1 , bleeding from the gingiva after irritation; value 2 , the presence of subgingival calculus; value 3 , shallow periodontal pockets (depth $4-5 \mathrm{~mm}$ ); and value 4 , deep periodontal pockets $(\geq 6 \mathrm{~mm})$. Bleeding upon irritation (value 1) indicated the need to improve oral hygiene, and the presence of subgingival calculus (value 2) indicated the need for scaling and professional oral hygiene. The presence of pathological periodontal pockets (values 3 and 4 ) required periodontal therapy, including professional oral hygiene. The CPITN was established on the basis of the highest values obtained during the examination with a calibrated periodontal probe.

We also determined the amount of SM in the stimulated saliva of the study cohort. Stimulated saliva was obtained by chewing paraffin gum for one minute and collecting the resulting saliva in a cup. The presence and amount of SM in the saliva samples were detected by a chairside test (Dentocult Strip Mutans, Orion Diagnostica $^{\mathrm{TM}}$ ). The detection of SM was based on the adhesion of the bacteria to test strips, which were added to a selective medium. The samples were incubated for 48 $\mathrm{h}$ at $37{ }^{\circ} \mathrm{C}$, and the amount of SM was evaluated according to the standardized table (Dentocult Strip Mutans). Degree 0 in the table indicates the presence of less than 10.000 CFU (colony forming units) in $1 \mathrm{ml}$ of saliva. Degree 1 indicates less than $100.000 \mathrm{CFU}$ in $1 \mathrm{ml}$ of saliva. Degrees 2 and 3 indicate 100.000 or more CFU in $1 \mathrm{ml}$ of saliva and were considered risk factors for dental caries development and transmission of SM to the oral cavities of infants after birth. The restorative dental treatment need was determined in cases of untreated caries.

\section{Statistical analysis}

The data obtained were analyzed statistically using Statgraphics software. The Mann-Whitney test, KruskalWallis test and chi-squared test were used; p-values less than 0.05 were considered statistically significant. A twotailed t-test was used for comparison of the two cohorts.

\section{Ethical considerations}

The study was approved by the Research Ethic Committee of The Faculty of Medicine in Pilsen, Charles University in Prague, the Czech Republic. 


\section{RESULTS}

\section{Characteristics of pregnant women}

The mean ages of the pregnant women in the study were $30.74 \pm 4.02$ years for the high-risk pregnancy group (R) and $30.76 \pm 3.46$ years for the control group $(\mathrm{F})$. The most serious conditions in the high-risk pregnancy group were gestational diabetes mellitus, pregestational diabetes, gynecological complications and systemic diseases (e.g. hypofunction of the thyroid gland, high blood pressure, cardiac disease and epilepsy) (Table 1).

Table 1. Pregnancy risk factors.

\begin{tabular}{lcc}
\hline Pregnancy risk factor & Number & $\%$ \\
\hline Gestational diabetes mellitus & 31 & 38.3 \\
Gynecological diseases & 23 & 28.4 \\
Systemic diseases & 16 & 19.8 \\
Pregestational diabetes mellitus & 7 & 8.6 \\
IVF & 3 & 3.7 \\
Age - related reasons & 1 & 1.2 \\
Total & 81 & 100.0 \\
\hline
\end{tabular}

Differences between the DMF indices of women in the highrisk pregnancy and control groups

The minimum DMF value in both groups was 1 , and the maximum values were 28 in group $\mathrm{R}$ and 27 in group F. The average DMF index value was 12.8 for group $\mathrm{R}$ and 11.5 for group $\mathrm{F}$ (Table 2); however, these differences in DMF values were not statistically significant $(P=0.1738)$.

Differences between the PBI values of women in the highrisk pregnancy and control groups

The minimum PBI value (value 0) was observed in one of the women in group $\mathrm{R}$ and one of the women in group $\mathrm{F}$. The maximum index value was 3.8 in group $\mathrm{R}$ and 2.9 in group F (Table 2). The differences in PBI values between the two groups were statistically significant $(P=0.0194)$.

Differences between the CPITN values of women in the high-risk pregnancy and control group

The CPITN values obtained are listed in Table 2. No statistically significant differences were found between the CPITN values of groups $\mathrm{R}$ and $\mathrm{F}(P=0.1139)$.

Differences between the amounts of SM in the saliva of women in the high-risk pregnancy and control groups

High levels of SM in the saliva (e.g., levels 2 and 3 of the standardized table of Orion Diagnostica ${ }^{\mathrm{TM}}$ ) were identified in 57 of the women in group $\mathrm{R}(70.4 \%)$ and 40 of the women in group $\mathrm{F}(65.5 \%)(P=0.39)$ (Table 3$)$.

Table 2. DMF, PBI and CPITN index.

\begin{tabular}{|c|c|c|c|c|c|c|c|c|}
\hline \multirow[t]{2}{*}{ Index DMF } & \multicolumn{2}{|c|}{ Pregnancy } & \multirow[t]{2}{*}{ Index PBI } & \multicolumn{2}{|c|}{ Pregnancy } & \multirow[t]{2}{*}{ Index CPITN } & \multicolumn{2}{|c|}{ Pregnancy } \\
\hline & $\mathrm{R}$ & $\mathrm{F}$ & & $\mathrm{R}$ & $\mathrm{F}$ & & $\mathrm{R}$ & $\mathrm{F}$ \\
\hline Minimum & 1 & 1 & Minimum & 0 & 0 & 0 & 0 & 1 \\
\hline Maximum & 28 & 27 & Maximum & 3.8 & 2.9 & 1 & 20 & 20 \\
\hline SD & 6.02 & 5.62 & SD & 0.9 & 0.74 & 2 & 43 & 34 \\
\hline \multirow[t]{3}{*}{$P$-value } & \multicolumn{2}{|c|}{0.1738} & $P$-value & \multicolumn{2}{|c|}{0.0194} & 3 & 16 & 5 \\
\hline & & & & & & 4 & 2 & 1 \\
\hline & & & & & & $P$-value & & \\
\hline
\end{tabular}

Table 3. The amounts of SM in the saliva.

\begin{tabular}{cccccc}
\hline SM in saliva & \multicolumn{5}{c}{ The course of pregnancy } \\
& R number & R \% & F number & F \% & $\Sigma$ \\
\hline 0 & 8 & 9.8 & 5 & 8.2 & 13 \\
1 & 16 & 19.7 & 16 & 26.2 & 32 \\
2 & 30 & 37.2 & 15 & 24.6 & 45 \\
3 & 275 & 33.3 & 25 & 41.0 & 52 \\
\hline$\Sigma$ & 81 & 100.0 & 61 & 100.0 & 142 \\
\hline
\end{tabular}


Differences in the dental restorative treatments needed by women in the high-risk pregnancy and control groups

We identified 62 women in group R (77\%) and 32 women in group $\mathrm{F}(52 \%)$ who required restorative dental treatments. The difference in treatment requirements between the two groups was statistically significant $(P=0.0027)$.

\section{DISCUSSION}

Dental caries and periodontal diseases are important oral conditions affecting many pregnant women. These conditions may be risk factors for pregnancy complications, preterm delivery, low birth weight and early childhood caries ${ }^{5-8}$. In this study, we examined the occurrence of dental caries, gingivitis and periodontitis in 61 women with normal pregnancies and 81 women with high-risk pregnancies.

The group of women with high-risk pregnancies was primarily composed of women with gestational diabetes mellitus and gynecological diseases. Gestational diabetes mellitus is defined as any degree of carbohydrate intolerance that occurs for the first time during pregnancy ${ }^{11}$. This disease is the most common metabolic disorder in pregnancy and can have serious consequences for both the mother and the development of the fetus ${ }^{12,13}$. The most common complications that occur immediately after birth are a birth weight greater than $4000 \mathrm{~g}$, hyperbilirubinemia, hypoglycemia, hypocalcemia and coagulation disorders. Higher hemoglobin levels and an increased risk of thrombosis and obstetric trauma related to the size of the fetus have also been described. Complications during childhood, including the development of brain dysfunctions and obesity, and complications in adulthood, including the development of type 2 diabetes mellitus, can also occur. Defects in hard dental tissue mineralization and problems during dental examination and treatment of children with brain dysfunctions can be risk factors for dental decay. Gestational diabetes can also result in complications for the pregnant women. It increases the risk of polyhydramnion, hypertension, recurrent urogenital infections, Caesarean delivery, birth injury and the persistence of diabetes after pregnancy or its return during a subsequent pregnancy ${ }^{13}$. Gestational diabetes has been associated with periodontal disease in a cross-sectional study ${ }^{13}$ and a case-control study ${ }^{14}$.

The most common periodontal disease in pregnancy is gingivitis ${ }^{15}$. Women who experience premature deliveries of infants with low-birth weights have been found to have significantly more severe and extended gingival inflammation than women with normal pregnancies ${ }^{16}$. A similar situation was found in our study. Statistically significant lower PBI index values were observed in women with normal pregnancies, but gingivitis was present in both groups of pregnant women in our study.

Periodontitis was evaluated using the CPITN index. The CPITN index is an epidemiological index and does not provide exact information on disease severity. This index was used because it is part of preventive recalls of all patients in the Dentistry Department in Pilsen.

Periodontitis may represent a risk factor for pregnancy complications, premature birth and low-birth weight ${ }^{7,17}$. It has been suggested ${ }^{5}$ that the effect of periodontal disease on low birth weight could result from the stimulation of fetal membranes with products derived from the inflamed periodontal tissues. Currently, the influence of the mother's periodontal disease status on her newborn's low birth weight is unclear ${ }^{18}$. High CPITN index values of 3 and 4 were detected in 6 women $(9.8 \%)$ in the normal pregnancy group and 18 women $(22.1 \%)$ in the high-risk pregnancy group. The CPITN index values in our cohort of women with high-risk pregnancies were more serious than those found in a retrospective study of women with pregnancy complications in Helsinki ${ }^{9}$.

Dental caries is the second most important disease of the oral cavity in pregnancy. The DMF index indicates the status of the oral cavity with regard to the number of teeth with decay, with fillings and extracted for caries. The mean DMF index score was 11.3 for the women with normal pregnancies and 12.8 for the women with high-risk pregnancies. Untreated dental caries was found in $41.3 \%$ of the women in group $\mathrm{F}$ and $51.8 \%$ of the women in group R. The presence of dental caries during pregnancy and especially after birth represents an increased risk of early childhood caries development. Cariogenic bacteria can be transmitted through the saliva to the oral cavities of infants. Factors affecting bacterial transmission are the levels of these microbes in the mother's saliva, the frequency and efficiency of transmission and the child's receptivity to bacterial colonization. Additional risk factors for the occurrence of early childhood caries include the timing of transmission, the child's age, the composition and flow of the child's saliva and the amount of carbohydrates in the diet ${ }^{12,19-21}$. In our study, high levels of SM were identified in the saliva of 40 women $(63.6 \%)$ in the normal pregnancy group and 57 women $(70.5 \%)$ in the high-risk pregnancy group. In contrast, Brambilla et al. ${ }^{22}$ found that only $21 \%$ of the pregnant women in their study had high levels of SM in their saliva. Another study of the association between cariogenic oral bacteria and preterm birth found high salivary levels of SM in 34\% of pregnant women $^{23}$. Restorative treatment of dental caries was necessary in $52.5 \%$ of the women in the normal pregnancy group and $66.2 \%$ of the women in the high-risk pregnancy group. Based on these findings, every pregnant woman should undergo two complete dental examinations for the prevention and treatment of dental caries and periodontal tissue diseases. During the dental appointment, it is necessary to remove calculus and to provide instructions on prenatal and postnatal nutrition with respect to maintaining proper oral hygiene and preventing caries. Pregnant women visiting dentists also have the opportunity to be informed about the dangers of transmitting Streptococcus mutans to the oral cavities of their infants. 


\section{CONCLUSIONS}

In this study, we focused on the oral health status of women with normal and high-risk pregnancies. Women with high-risk pregnancies showed increased values in all measured indices and tests (DMF index, PBI index, CPITN index and the amounts of Streptococcus mutans in the saliva). Statistically significant differences in the PBI index and the need for dental treatment were found between women with normal and high-risk pregnancies. Women with complications during pregnancy had severe gingivitis and needed more frequent dental treatment than did women with normal pregnancies. This study supports the hypothesis of an association between oral health and the course of pregnancy. Based on our findings, women with high-risk pregnancies deserve additional attention during preventive dental examinations, and it is necessary to motivate them to take particularly good care of their oral health.

\section{ACKNOWLEDGEMENT}

This study was supported by IGA NS/ 9732 - 4 of the Health Ministry of the Czech Republic.

\section{CONFLICT OF INTEREST STATEMENT}

Author's conflict of interest disclosure: The authors stated that there are no conflicts of interest regarding the publication of this article.

\section{REFERENCES}

1. Bogges KA, Edelstein BL. Oral Health in Women During Preconception and Pregnancy: Implications for Birth Outcomes and Infant Oral Health. Matern Child Health J 2006;10:169-74.

2. Berkovitz RJ. Causes, Treatment and Prevention of Early Childhood Caries: A Microbiologic Perspective. J Can Dent Assoc 2003;69:304-7.

3. Agueda A, Ramón JM, Marnau C, Guerrero A, Echeveria JJ. Periodontal disease as a risk factor for adverse pregnancy outcomes: a prospective cohort study. J Clin Periodontol 2008;36:16-22.
4. Xiong X, Buekens P, Fraser WD, Beck J, Offenbacher S. Periodontal disease and adverse pregnancy outcomes: a systematic review. BJOG 2006;113:135-43.

5. Offenbacher S, Katz V, Fertik G, Collins J, Boyd D, Maynor G et al. Periodontal infection as a possible risk factor for preterm low birth weight. J Periodontol 1996;67:1103-13.

6. Bobetsis YA, Barros SP, Offenbacher S. Exploring the relationship between periodontal disease and pregnancy complications. J Am Den Assoc Suppl 2.,2006;137:7-13.

7. Jeffcoat MK, Geurs N, Reddy MS, Cliver SP, Goldenberg RL, Hauth JC. Periodontal infection and pretem birth. J Amer Dent Assoc 2001;132:875-80.

8. Marin C, Segura-Egea JJ, Martínez-Sahuquillo A, Bullón P. Correlation between infant birth weight and mother's periodontal status. J Clin Periodontol 2005;32:299-304.

9. Meurman JH, Furuholm J, Kaaja R, Rintamäki R, Tikkanen U. Oral health in women with pregnancy and delivery complications. Clin Oral Invest 2006;10:96-101.

10. WHO. Oral health surveys. Basic Methods. 4th ed. Geneva 1997.

11. Definition, Diagnosis and Classification of Diabetes Mellitus and its Complications. Geneva WHO 1999.

12. Setji LT, Brown AJ, Feinglos MN. Gestational Diabetes Mellitus. Clin Diabet 2005;23:17-24.

13. Xiong $X$, Buekens $P$, Vastardis S, Pridjan G. Periodontal disease and gestational diabetes mellitus. AJOG 2006;195:1086-9.

14. Xiong X, Elkind-Hirsch KE, Vastardis S, Delarosa RL, Pridjian G, Buekens P. Periodontal Disease Is Associated With Gestational Diabetes Mellitus: A Case - Control Study. J Periodontol 2009;80:1742-9.

15. Vasiliauskiene I, Milciuviene S, Bendoraitiene E, Narbutaite J, Slabsinskiene E, Andruskeviciene V. Dynamics of Pregnant Women's Oral Health Status during Preventive Programme. Stomatologija 2007;9:129-36.

16. López NJ, Smith PC, Gutierrez J. Higher Risk of Preterm Birth and Low Birth Weight in Women with Periodontal Disease. J Dent Res 2002;81:58-63.

17. Moliterno LFM, Monteiro B, da Silva Figueredo CM, Fischer RG Association between periodontitis and low birth weight: a case control study. J Clin Periodontol 2005;32:886-90.

18. Vettore MV et al. The Relationship between Periodontitis and Preterm Low Birthweight. J Dent Res 2008;87:73-8.

19. Caufield PW, Cutter GR, Dasanazke AP. Initial acquisition of mutans streptococci by infants: evidence for a discrete window of infectivity. J Dent Res 1993;72:37-45.

20. Slavkin HC. Streptococcus mutans, early childhood caries and new opportunities. J Amer Dent Assoc 1999;130:1787-92.

21. Weerheijm KL, Uyttendaele BFM, Euwe HC, Groen HJ. Prolonged demand breastfeeding and nursing caries. Car Res 1998;32:46-50.

22. Brambilla E, Felloni A, Cagliani M, Malerba A, Garcia - Godoy, Strohmenger Z. Caries prevention during pregnancy: results of a 30 - month study. J Am Dent Assoc. 1998; 129:871-77.

23. Durand R, Gunselman EL, Hodges JS, DiAngelis AJ, Michalowicz BS. A pilot study of the association between cariogenic oral bacteria and preterm birth. Oral Diseases 2009;15:400-6. 